\title{
Development of laser-induced breakdown spectroscopy for analyzing deposited layers in ITER
}

\author{
J Karhunen ${ }^{1}$, A Hakola ${ }^{1}$, Likonen ${ }^{1}$, A Lissovski ${ }^{2}$, P Paris ${ }^{2}$, M Laan ${ }^{2}$, K Piip $^{2}$, C Porosnicu ${ }^{3}$, C P \\ Lungu $^{3}$, K Sugiyama ${ }^{4}$ \\ ${ }^{1}$ VTT Technical Research Centre of Finland, Association Euratom-Tekes, 02044 VTT, Finland \\ ${ }^{2}$ Institute of Physics, University of Tartu, 51010 Tartu, Estonia \\ ${ }^{3}$ INFLPR, MEdC EURATOM Association Magurele-Bucharest, 077125, Romania \\ ${ }^{4}$ Max-Planck-Institut für Plasmaphysik, EURATOM Association, D-85748 Garching, Germany
}

\begin{abstract}
Laser-induced breakdown spectroscopy (LIBS) experiments on different ITER-relevant berylliumtungsten mixtures were performed to develop LIBS towards tokamak applications. Some of the samples were doped or implanted with deuterium to simulate fuel retention. The results indicate that beryllium and tungsten have a number of distinguishable spectral lines for diagnostics purposes, but detection of deuterium is challenged by its low concentration and low fluences used. By studying the depth profiles of the coatings, the removal rates of the layers were observed to depend on the thickness, composition, and deuterium content of the coating. Quantitative studies of the compositions were made by CF-LIBS, and the results agreed well with those given by ion-beam methods.
\end{abstract}

\section{Introduction}

In future fusion reactors, the lifetime of plasma-facing components can be shortened and retention of fuel into them significantly increased by the formation of different mixed deposited layers [1]. Thereby, the first walls of the reactors should be actively monitored, also in between plasma discharges to determine the locations, thicknesses, and compositions of the deposited layers.

Laser-induced breakdown spectroscopy (LIBS) is a potential method for these kinds of in situ studies. LIBS is based on powerful laser pulses ejecting material from the surface of the studied sample, which leads to the formation of a plasma plume. The light emitted by the plume is recorded and the composition of the sample is determined from the measured spectra by identifying the observed spectral lines and comparing them to the emission spectra of different elements [2]. In this contribution, the feasibility of LIBS to analyze ITER-relevant material mixtures has been studied with the purpose of developing LIBS to be applicable with the ITER-like wall at JET, and eventually in ITER. The results are compared to those obtained by the standard ion-beam methods secondary ion mass spectrometry (SIMS), Rutherford backscattering spectroscopy (RBS), and nuclear reaction analysis (NRA).

\section{Experimental system}

The experiments were carried out using a set-up specifically built for LIBS studies of berylliumcontaining samples as schematically shown in figure 1 . The samples were located inside a vacuum chamber which was pumped to a base pressure of $10^{-7}$ mbar. A pulsed Nd:YAG laser, operating at $1064 \mathrm{~nm}$ and producing 5-ns laser pulses with Gaussian energy profiles (maximum energy $50 \mathrm{~mJ}$ ), was used to produce the LIBS plasma. The laser beam was guided into the chamber by a set of 
mirrors and focused on the surface of the sample by a system of two lenses with focal lengths of 150 $\mathrm{mm}$ and $350 \mathrm{~mm}$, resulting in fluences up to $3 \mathrm{Jcm}^{-2}$. The emission spectra were recorded perpendicular to the laser beam with either a low-resolution $(0.63 \mathrm{~nm})$ Avantes Avaspec 2048 USB2 spectrometer or a high-resolution $(0.04 \mathrm{~nm})$ Andor SR-750 spectrometer equipped with an Andor iStar 340T ICCD camera. In the latter case, the delay between the laser pulse and the onset of recording, as well as the width of the measuring gate, could be varied to optimize the widths of the spectral lines and the intensity level of the background continuum.

Various 500 - 2500-nm thick coatings of pure beryllium or different mixtures of beryllium and tungsten were produced for the experiments by the thermoionic vacuum arc (TVA) method [3]. The ratio of beryllium and tungsten (in at.\%) in the mixed layers varied from 75:25 to 95:5. The coatings were deposited on either round stainless-steel substrates with a diameter of $25 \mathrm{~mm}$ or rectangular silicon substrates of $12 \times 15 \mathrm{~mm}^{2}$. The substrate materials were selected for practical reasons, but the results are not dependent on the substrate materials. Thereby the conclusions drawn here will be valid also for ITER with layers deposited on either beryllium or tungsten. To study fuel retention, part of the samples was doped with deuterium during their deposition. In addition, some of the non-doped samples were implanted with 200-eV deuterons at IPP Garching to simulate implantation of energetic ions in a tokamak.

\section{Detectability of spectral lines}

Beryllium was observed to have four intense spectral lines suitable for diagnostics purposes: atomic Be(I) lines at $332 \mathrm{~nm}$ and $457 \mathrm{~nm}$ and ionic Be(II) lines at $313 \mathrm{~nm}$ and $467 \mathrm{~nm}$. By optimizing the measuring delay, the width of the beryllium lines as well as the background continuum were successfully minimized, as can be seen in figure 2a. As shown in figure $2 \mathrm{~b}$, the most intense tungsten lines are also narrow and clearly detectable, but their intensity is lower, leading to a worse signal-tonoise ratio than for beryllium.

Considering the detection of deuterium in the implanted and doped samples, the $\mathrm{H}_{\alpha}$ line at $656 \mathrm{~nm}$ was observed only with the first laser pulse - and even then its origin is most probably hydrogen contamination from air. In the case of doped samples, the poor detectability can be explained by their low deuterium content ( $\sim 0.01$ at.\%), as suggested by NRA and SIMS. However, this is not the case for the implanted samples: a clear deuterium signal was detected by SIMS in each implanted sample for which the NRA results indicate D contents above 0.1 at.\%. In pure beryllium, the implantation depth was approximately $300 \mathrm{~nm}$, whereas the signal existed throughout the mixed beryllium-tungsten coatings independent of their thicknesses.

Detection of deuterium can be affected by the laser parameters. The fluence used in these studies was rather low so that deuterium atoms may have been insufficiently excited; preliminary results with a renewed experimental system indicate better detectability of hydrogen with higher fluences. In addition, the heating effect of infrared laser pulses may have led to thermal desorption of the implanted deuterium already after the first laser shot [4]. This explains why the $\mathrm{H}_{\alpha}$ line was never observed beyond the first pulse - even if the amount of deuterium had been sufficient to exceed the detection limit of LIBS. The restrictions could be avoided by using an ultraviolet laser instead of an infrared one, since with UV pulses the laser-matter interaction is restricted closer to the sample surface than in the case of IR pulses, and the heating effect is thus not as widely spread. The experimental set-up is currently being renewed to enable this.

\section{Depth profiles}


To investigate how quickly different mixed layers are removed in real applications, the depth profiles of the coatings were determined by studying the integrated intensities of atomic and ionic lines of beryllium at $457 \mathrm{~nm}$ and $467 \mathrm{~nm}$, respectively, and an atomic line of iron from the substrate at $337 \mathrm{~nm}$ as functions of the number of the laser shots. Typical results for a pure beryllium coating and a beryllium-tungsten mixture of different thicknesses are presented in figure 3, where the intensities are normalized to range from 0 to 1 . In the depth profiles, the coating is represented by a plateau regime whose width depends on the thickness and ablation rate of the layer.

The ablation rates of the coatings were determined by finding the $\mathrm{e}^{2}$-folding widths of the depth profiles and by dividing the thicknesses of the layers by the corresponding shot number. Here, the factor $\mathrm{e}^{2}-$ a characteristic value for laser beams with a Gaussian energy distribution - was selected to represent the state where removal of the coating is induced only by the very edges of the laser beam.

Generally, the ablation rates of different coatings ranged within $15-40 \mathrm{~nm} / \mathrm{shot}$, averaging around 30 $\mathrm{nm} / \mathrm{shot}$. In the case of the pure beryllium coatings, the ablation rate was observed to increase as a function of the thickness of the coating, most probably due to inferior quality of the thick layers. The ablation rates of the mixed layers were slightly higher than those of pure beryllium, suggesting a weaker structure of the beryllium-tungsten compounds. A further increase in the ablation rates is caused by the lattice damages induced by the deuterium implantation. Hence, the ablation rate of a deposited layer is dependent on its thickness, composition, and deuterium content. A large variety of calibration measurements is thus required for resolving the absolute depth profiles of the mixed layers in tokamak applications.

\section{Quantitative studies by calibration-free LIBS}

The concentrations of beryllium and tungsten were determined quantitatively for the Be-W sample in figure 3b using the calibration-free LIBS (CF-LIBS) method [5]. CF-LIBS relies on determining the temperature of the plasma from a Saha-Boltzmann plot [6] drawn for each emitting species in the plasma plume. In these studies, seven atomic and three ionic lines of beryllium and 27 atomic and 12 ionic lines of tungsten within the wavelength range 253-490 nm were chosen for the analyses. The studies were made using 20 consecutive laser pulses, corresponding to the topmost $650 \mathrm{~nm}$ of the 1790-nm thick layer.

The Saha-Boltzmann plots suggested plasma temperatures of $12400-12800 \mathrm{~K}$, varying from pulse to pulse. By extracting the corresponding partition functions from the NIST database [7], the concentration profiles in figure 4 were obtained. In the figure, the concentrations determined by CFLIBS are compared to those given by RBS, and the shapes of the profiles to the SIMS profiles of the elements in question.

The concentrations given by CF-LIBS and RBS agree well except for the approximately 150-nm thick layer just below the surface of the sample. The difference may be explained with the presence of oxygen in oxidized beryllium and tungsten, since the spectral line of atomic oxygen at $777 \mathrm{~nm}$ was detected during the first five laser pulses, corresponding to $150 \mathrm{~nm}$ in depth. For the studied sample, the oxygen concentration was not determined beforehand, but RBS studies of other samples from the same set suggest oxygen concentrations as high as $10-30 \%$ in the topmost layer. However, due to overlapping of oxygen and substrate silicon peaks in the RBS spectrum, the error of this estimate is rather large.

Oxygen is not taken into account when calculating the concentrations by either CF-LIBS or RBS in these studies. Instead, CF-LIBS considers beryllium oxide as pure beryllium, and RBS ignores both 
oxygen and oxidized beryllium. Hence, CF-LIBS overestimates the beryllium content close to the surface, whereas RBS underestimates it, which explains why the increase in the CF-LIBS data is larger than that observed by SIMS. Since in these studies both CF-LIBS and RBS regard the sum of concentrations of beryllium and tungsten as unity, the described distorted behaviour appears mirrored in the tungsten profiles.

Although CF-LIBS appears to agree with RBS on the concentration profiles, the results should not be considered definite. Reliable results require elimination of self absorption either by neglecting the self-absorbed spectral lines or by applying a correction algorithm to remove the effect of self absorption. The algorithm presented in [8] used in these studies depends heavily on the choice of specific reference lines, whose self absorption is assumed to be negligibly small; in reality, this condition may not be satisfied. Moreover, due to the small fluence used in these studies, the intensities of the weaker lines of beryllium and tungsten - including the reference lines - were very low, which makes the effect of the background noise noticeable. Choosing different combinations of the reference lines for the emitting species led to variations in the Saha-Boltzmann plots which have a great impact on the values of the plasma temperature and concentrations given by CF-LIBS. Further studies are required to confirm the choice of the reference lines and to enable proper correction of self absorption.

\section{Conclusions}

Different ITER-relevant compositions of beryllium and tungsten were studied by LIBS to develop the method for in situ monitoring of mixed deposited layers on the plasma-facing surfaces of future fusion reactors. Some of the samples were doped or implanted with deuterium to study fuel retention.

The studies show that beryllium has a number of clearly detectable spectral lines that can be used for diagnostics purposes. Deuterium, however, could not be reliably detected, although its presence in the implanted samples was verified by SIMS. This is due to low deuterium concentrations in the samples, low fluence of the laser beam, and thermal desorption of deuterium. By studying the intensities of beryllium lines as functions of the number of laser pulses, smooth and consistent depth profiles were obtained. Based on these depth profiles, the ablation rate of mixed beryllium-tungsten coatings appeared to be larger than that of pure beryllium and further increased by deuterium implantation.

The quantitative composition of a beryllium-tungsten mixture was determined by CF-LIBS, and the results agreed well with those given by RBS. Differences between the two methods as well as deviations from the shape of the SIMS profile were observed within the topmost $150 \mathrm{~nm}$ of the studied coating, probably due to presence of oxygen in the layer. Further studies are still required to confirm the CF-LIBS results.

After the studies presented in this contribution, the experimental set-up has been partly renewed. The laser has been replaced with a more powerful one, providing fluences up to $40 \mathrm{Jcm}^{-2}$ and enabling LIBS studies also with visible and ultraviolet laser wavelengths, which leads to more reliable detection of deuterium: higher fluences have been observed to increase the $\mathrm{H}_{\alpha}$ signal, and thermal desorption of deuterium is expected to be reduced with UV pulses.

\section{References}

[1] R A Pitts et al., Plasma Physics and Controlled Fusion 47 (2005) B303—B322

[2] R E Russo et al., Talanta 57 (2002) 425—451

[3] C P Lungu et al., Vacuum 76 (2004) 127-130 
[4] M. Oberkofler et al., Journal of Nuclear Materials 415 (2011) S724—S727

[5] A Ciucci et al., Applied Spectroscopy 53 (1999) 960—964

[6] E Tognoni et al., Spectrochimica Acta Part B 62 (2007) 1287-1302

[7] NIST Atomic Spectra Database (ver. 5.0), http://physics.nist.gov/asd

[8] L Sun et al., Talanta 79 (2009) 388—395

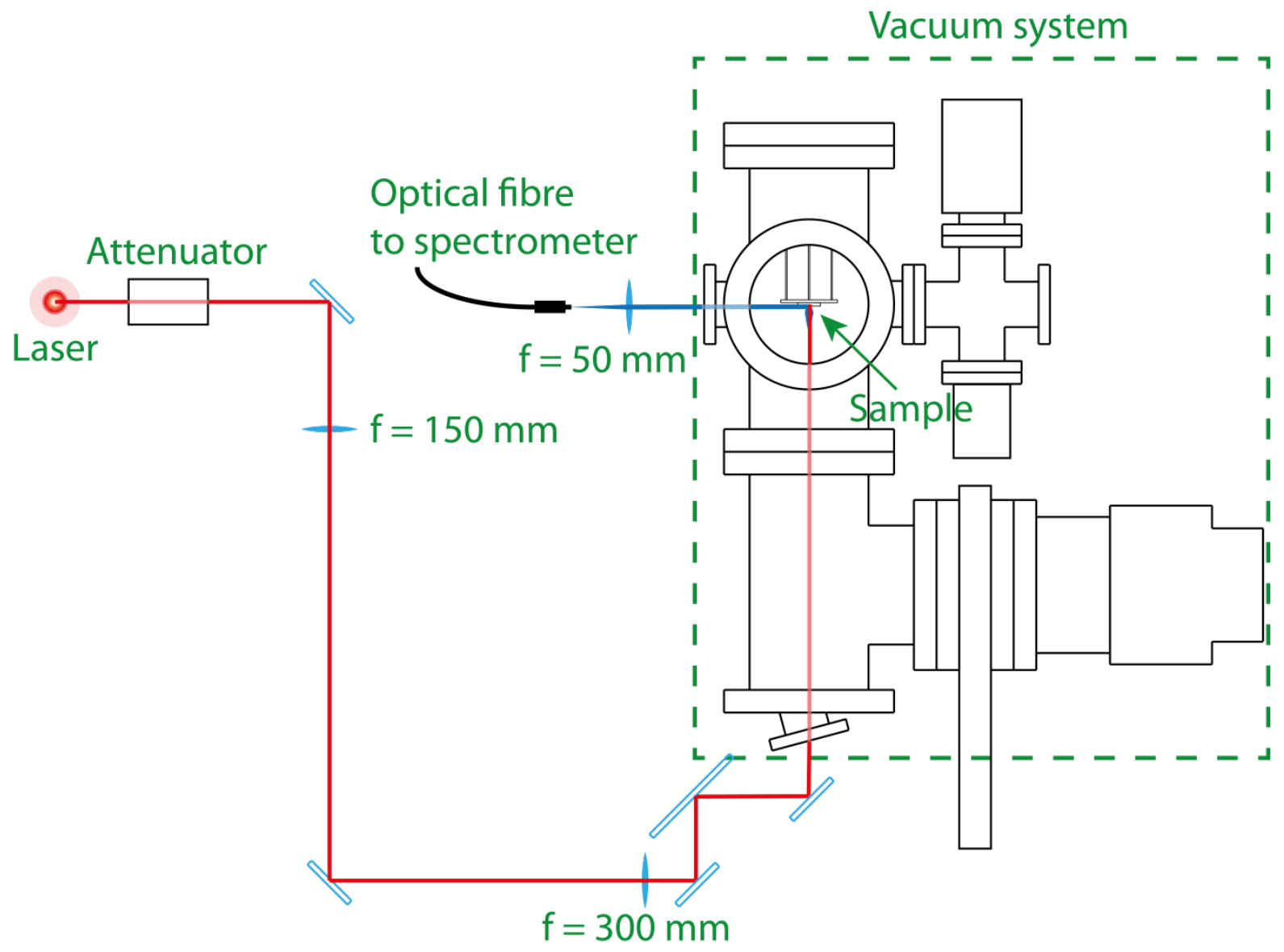

Figure 1: Experimental system used in the studies.
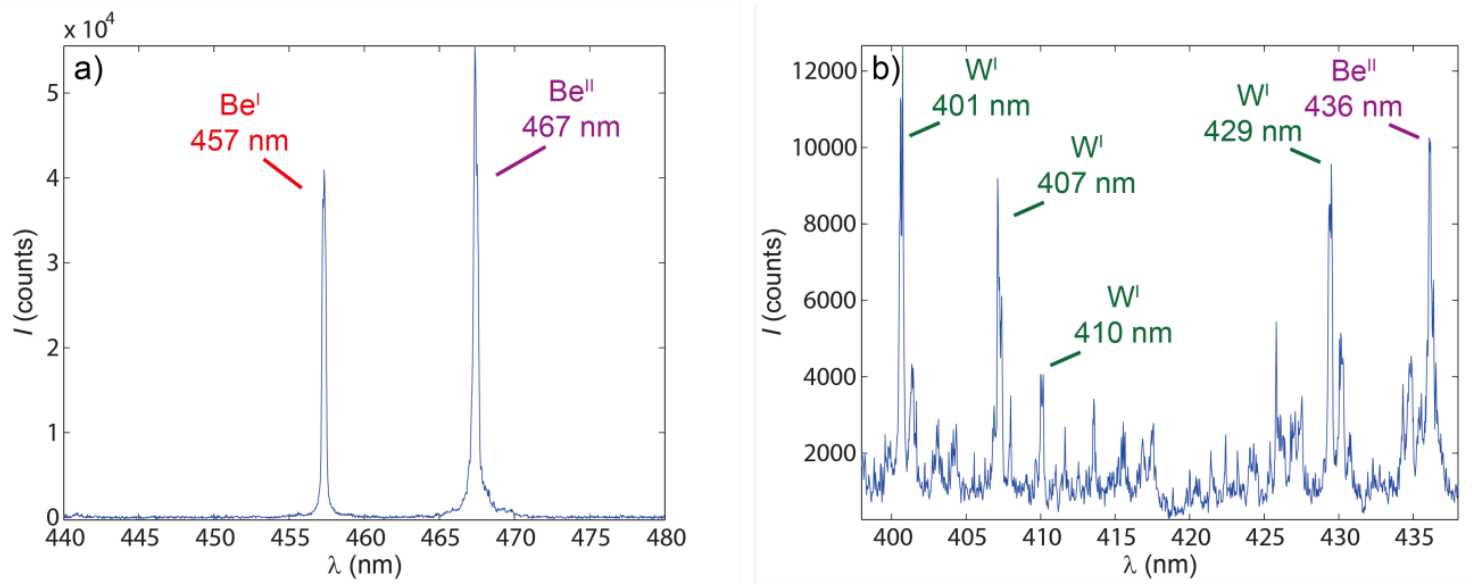

Figure 2: Most intense spectral lines of a) beryllium and b) tungsten. 


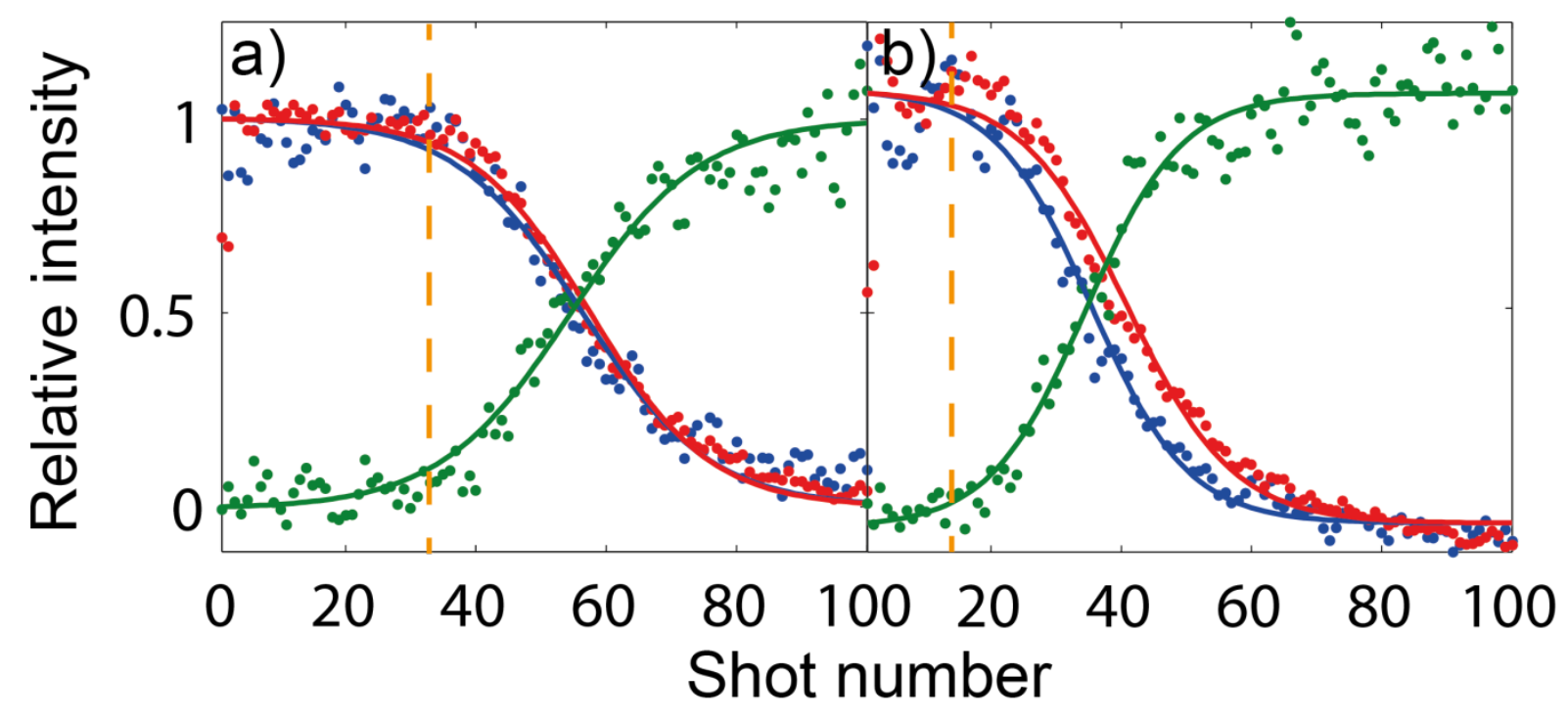

- $\operatorname{Be}(\mathrm{I}) 457 \mathrm{~nm} \cdot \mathrm{Be}(\mathrm{II}) 467 \mathrm{~nm} \cdot \mathrm{Fe}(\mathrm{I}) 337 \mathrm{~nm}$

Figure 3: Normalized depth profiles of a) a 2340-nm thick pure beryllium coating and b) a 1790-nm thick coating with a Be-W ratio of approximately 80:20. The interface between the coating and the substrate is presented by the orange dashed line.

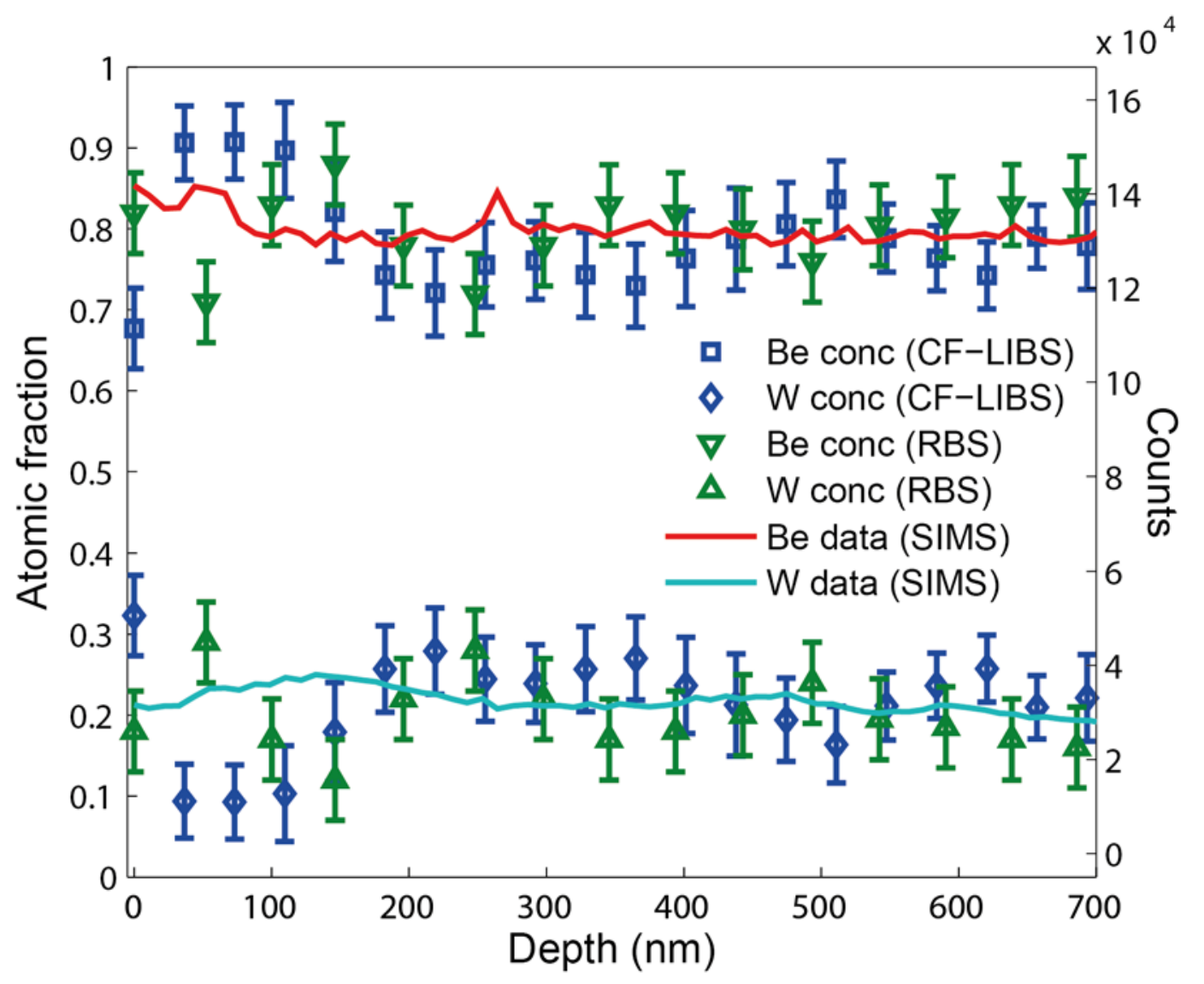

Figure 4: Concentration profiles given by CF-LIBS and RBS (left axis) and SIMS depth profiles (right axis). 